\title{
Uniform approximation for period-quadrupling bifurcations
}

\author{
Martin Sieber $\dagger$ and Henning Schomerus $\ddagger$ \\ $\dagger$ Abteilung Theoretische Physik, Universität Ulm, D-89069 Ulm, Germany \\ $\ddagger$ Fachbereich Physik, Universität-Gesamthochschule Essen, D-45117 Essen, Germany
}

Received 26 August 1997

\begin{abstract}
We derive a uniform approximation for semiclassical contributions of periodic orbits to the spectral density which is valid for generic period-quadrupling bifurcations in systems with a mixed phase space. These bifurcations involve three periodic orbits which coalesce at the bifurcation. In the vicinity of the bifurcation the three orbits give a collective contribution to the spectral density while the individual contributions of Gutzwiller's type would diverge at the bifurcation. The uniform approximation is obtained by mapping the action function onto the normal form corresponding to the bifurcation. This article is a continuation of previous work in which uniform approximations for generic period- $m$-tupling bifurcations with $m \neq 4$ were derived.
\end{abstract}

\section{Introduction}

Semiclassical approximations for the density of states of a quantum system can be expressed in terms of classical periodic orbits [1-5]. The form in which the periodic orbits contribute in these approximations is not unique; it rather depends on the characteristics of the classical motion. Up until now, complete approximations in terms of periodic orbits have been derived only for the cases of either integrable or globally chaotic classical motion. In the more general situation of a mixed phase space, difficulties arise due to bifurcations, i.e. because of the coalescence of two or more periodic orbits as the energy or some external parameter is varied. In this article we describe the treatment of a generic type of bifurcation. We derive a uniform approximation for the joint contribution of orbits that participate in a period-quadrupling bifurcation.

One way to derive semiclassical approximations is by starting from Feynman's path integral and evaluating all integrals semiclassically. The periodic-orbit contributions then arise from the stationary points of oscillatory integrals. If the periodic orbits are isolated the integrals can be evaluated by a stationary-phase approximation and one obtains Gutzwiller's trace formula. At bifurcations of periodic orbits, however, different stationary points coalesce and the stationary-phase approximation breaks down. This is because the different stationary points cannot be treated separately in the vicinity of the bifurcation. Instead one has to treat them collectively. This is done in terms of certain canonical integrals with the same structure of stationary points.

The characteristic arrangement of stationary points in the vicinity of a bifurcation is described by its normal form. The generic normal forms for bifurcations in autonomous systems with two degrees of freedom or, equivalently, two-dimensional area-preserving maps have been classified by Meyer and Bruno [6-8]. They depend on the ratio $m$ of the 
primitive periods of the periodic orbits which coalesce at the bifurcation. The corresponding bifurcations are named period- $m$-tupling bifurcations. Ozorio de Almeida and Hannay [9] derived transitional (or local) approximations for the contributions of periodic orbits near generic bifurcations. These approximations are expressed in terms of canonical catastrophe diffraction integrals. They are transitional approximations because they are valid in the vicinity of the bifurcation. Further from the bifurcation, however, they do not yield the correct amplitudes in Gutzwiller's approximation for the contribution of isolated periodic orbits.

In two previous articles we extended the results of Ozorio de Almeida and Hannay [9] by deriving uniform approximations which interpolate between the transitional approximation at the bifurcation and Gutzwiller's approximation for isolated periodic orbits $[10,11]$ (see also [12]). These uniform approximations were obtained by including higher-order corrections to the normal form expansion and then simplifying the integrals by appropriate coordinate transformations. The derivations were completed for the cases $m>4$ and $m<4$, respectively. In this paper we treat the remaining case $m=4$. This case is more complicated than the others since it involves three periodic orbits whose action differences all increase with the same power of the parameter that describes the distance to the bifurcation. We use a different method for the derivation of the uniform approximation than was previously used. We apply techniques of catastrophe theory for obtaining uniform approximations for oscillatory integrals with almost coincident stationary points by performing a mapping onto the normal form [13-15]. The motivation is to provide a more elegant derivation; the results are, of course, the same for both approaches.

In the following section we present the uniform approximation that is derived in appendix A and discuss several limiting cases. The result is given for autonomous systems with two degrees of freedom and for two-dimensional area-preserving maps. We apply the uniform approximation numerically to the kicked top and discuss the limits of its validity.

\section{The uniform approximation}

The semiclassical contributions of periodic orbits to the spectral density can be obtained by expressing the density in terms of the trace of the (retarded) Green function,

$$
d(E)=\sum_{n} \delta\left(E-E_{n}\right)=-\frac{1}{\pi} \operatorname{Im} \operatorname{Tr} G(E)
$$

and evaluating the trace semiclassically in the vicinity of the orbits. If the Green function is expressed in a mixed coordinate-momentum representation and the integrals over the components of the coordinates along the periodic orbits are carried out one arrives at an integral expression of the form

$$
\begin{aligned}
& d_{\xi}(E) \approx \frac{1}{2 \pi^{2} \hbar^{2}} \operatorname{Re} \int_{-\infty}^{\infty} \mathrm{d} q^{\prime} \int_{-\infty}^{\infty} \mathrm{d} p \frac{1}{r} \frac{\partial \hat{S}}{\partial E}\left|\frac{\partial^{2} \hat{S}}{\partial p \partial q^{\prime}}\right|^{\frac{1}{2}} \\
& \times \exp \left[\frac{\mathrm{i}}{\hbar} \hat{S}\left(q^{\prime}, p, E\right)-\frac{\mathrm{i}}{\hbar} q^{\prime} p-\frac{\mathrm{i} \pi}{2} \nu\right] .
\end{aligned}
$$

For a more detailed derivation of this integral see $[10,11]$. In (2) the origin of the coordinate system is located on a central periodic orbit with repetition number $r$. Furthermore, $p$ and $q^{\prime}$ are coordinates in a Poincaré surface of section perpendicular to the orbit, and $\hat{S}\left(q^{\prime}, p, E\right)$ 
is the generating function for the $r$ th iterate of the Poincare map. It obeys the conditions

$$
\frac{\partial \hat{S}}{\partial q^{\prime}}=p^{\prime} \quad \frac{\partial \hat{S}}{\partial p}=q \quad \frac{\partial \hat{S}}{\partial E}=T
$$

where the primed quantities are the final coordinates, the unprimed quantities the initial coordinates, and $T$ is the time from initial to final point. The index $\xi$ of the spectral density denotes the contributions from a group of orbits in the vicinity of the central periodic orbit. The periodic orbits are the solutions of

$$
\frac{\partial \hat{S}}{\partial q^{\prime}}=p \quad \frac{\partial \hat{S}}{\partial p}=q^{\prime}
$$

and correspond to stationary points of the integral (2).

If the integral in (2) is evaluated in stationary-phase approximation one obtains Gutzwiller's contributions of isolated periodic orbits. For a periodic orbit labelled by $\gamma$ this contribution is given by

$$
d_{\gamma}(E)=\frac{A_{\gamma}(E)}{\pi \hbar} \cos \left(\frac{S_{\gamma}(E)}{\hbar}-\frac{\pi}{2} \nu_{\gamma}\right)
$$

where

$$
A_{\gamma}(E)=\frac{T_{\gamma}(E)}{r_{\gamma} \sqrt{\left|\operatorname{Tr} M_{\gamma}-2\right|}} .
$$

Here $S_{\gamma}, T_{\gamma}, r_{\gamma}, M_{\gamma}$ and $v_{\gamma}$ are, respectively, the action, period, repetition number, stability matrix, and Maslov index of the orbit.

In the vicinity of a bifurcation a stationary-phase evaluation of the integrals in (2) is not appropriate. Instead one has to integrate collectively over all stationary points which are involved in the bifurcation. This is done by inserting the normal form of the generating function $\hat{S}\left(q^{\prime}, p, E\right)$ for the considered bifurcation into (2). For a generic period-quadrupling bifurcation the repetition number $r$ is a multiple of 4; we denote in the following $l=r / 4$. The normal form for this case is given by

$$
\begin{aligned}
\hat{S}\left(q^{\prime}, p, E\right)= & S_{0}(E)+q^{\prime} p-\frac{\varepsilon}{2}\left(q^{\prime 2}+p^{2}\right)-\frac{a}{4}\left(q^{\prime 4}+2 p^{2} q^{\prime 2}+p^{4}\right) \\
& -\frac{b}{4}\left(q^{\prime 4}-6 p^{2} q^{\prime 2}+p^{4}\right) \\
= & S_{0}(E)+q^{\prime} p-\varepsilon I-a I^{2}-b I^{2} \cos (4 \Phi)
\end{aligned}
$$

where $p=\sqrt{2 I} \cos \Phi$ and $q^{\prime}=\sqrt{2 I} \sin \Phi$. The parameter $\varepsilon$ is zero at the bifurcation. The normal form (7) is obtained from an expansion of the Hamiltonian in the vicinity of the central periodic orbit, and $S_{0}$ is the action of this orbit.

The generic period-quadrupling bifurcation that is described by the normal form (7) involves three periodic orbits, a central orbit and two satellite orbits. The bifurcation occurs in two different forms depending on the relative magnitude of the two coefficients $a$ and $b$ in (7). In the case $|a|<|b|$ there are two real orbits and one complex orbit before and after the bifurcation, the stable central orbit, an unstable satellite orbit and a complex satellite orbit. At the bifurcation one of the satellites becomes complex and the other becomes real. For $|a|>|b|$ the two satellite orbits are both complex on one side of the bifurcation and both real on the other, where one of them is stable and the other unstable. The central orbit is real and stable on both sides of the bifurcation.

In the vicinity of the bifurcation, i.e. for sufficiently small values of $\varepsilon$, the contribution of the orbits can be described by the transitional approximation of Ozorio de Almeida and 
Hannay [9]. It is obtained by approximating the pre-exponential factor in (2) by its value at the origin and evaluating the integral with the normal form (7) for the action. This yields the semiclassical contribution in terms of the diffraction catastrophe integral for the catastrophe $X_{9}$. Further from the bifurcation the transitional approximation splits up into a sum of separate contributions of Gutzwiller's type. However, in this limit the semiclassical amplitudes come out with fixed relationships which are, in general, not in accordance with the periods and stabilities of the orbits. In more detail, the approximation is good as long as the following relations between the monodromy matrices and the periods of the orbits hold,

$$
\begin{aligned}
& 4 \Delta S_{21} \operatorname{Tr} M_{0}+\Delta S_{20} \operatorname{Tr} M_{1}=8 \Delta S_{21}+2 \Delta S_{20} \\
& 4 \Delta S_{12} \operatorname{Tr} M_{0}+\Delta S_{10} \operatorname{Tr} M_{2}=8 \Delta S_{12}+2 \Delta S_{10} \\
& \Delta S_{20} \operatorname{Tr} M_{1}+\Delta S_{10} \operatorname{Tr} M_{2}=2 \Delta S_{20}+2 \Delta S_{10}
\end{aligned}
$$

where $\Delta S_{i j}=\left(S_{i}-S_{j}\right) / 2$ and $T_{0}=T_{1}=T_{2}$. The index 0 denotes the central orbit and the indices 1 and 2 the two satellite orbits. Only two of the three equations in (8) are independent. The relations (8) follow from the normal form (7) (cf equations (22) and (24) in appendix A). For a general system they are only valid in the vicinity of the bifurcation. With increasing $\varepsilon$ they lose their validity, and the transitional approximation gradually becomes inaccurate. In order to obtain a formula which uniformly interpolates over the region from the bifurcation up to regimes where Gutzwiller's approximation is valid (without restrictions on the semiclassical amplitudes) one has to consider two modifications. First, higher-order corrections to the normal form (7) cannot be neglected any more. However, one can apply a mapping which brings the exponent in (2) back to the normal form. Second, one has to take into account the differences of the values of the exponential prefactor in (2) at the different stationary points. These steps are carried out in appendix A. In the following we discuss the uniform approximation which is obtained there.

The uniform approximation for the joint semiclassical contribution of orbits which are involved in a generic period-quadrupling bifurcation is given by

$d_{\xi}(E) \approx \frac{1}{4 l \pi \hbar^{2}} \operatorname{Re} \int_{0}^{\infty} \mathrm{d} I\left[T_{0}+\alpha_{1} I+\alpha_{2} I^{2}\right] J_{0}\left(\frac{\tilde{b} I^{2}}{\hbar}\right) \exp \left[\frac{\mathrm{i}}{\hbar}\left(S_{0}-\tilde{\varepsilon} I-\tilde{a} I^{2}\right)-\frac{\mathrm{i} \pi}{2} \nu\right]$

where $J$ denotes the Bessel function of the first kind and

$$
\begin{aligned}
& \tilde{\varepsilon}=\frac{\sigma_{\tilde{\varepsilon}} T_{0}}{4 l A_{0}} \quad \tilde{a}=\frac{\tilde{\varepsilon}^{2}\left(\Delta S_{10}+\Delta S_{20}\right)}{16 \Delta S_{10} \Delta S_{20}} \quad \tilde{b}=\frac{\tilde{\varepsilon}^{2} \Delta S_{21}}{16 \Delta S_{10} \Delta S_{20}} \\
& \alpha_{1}=l \sigma_{\tilde{\varepsilon} \tilde{\varepsilon}} \tilde{\varepsilon}^{2}\left[\frac{\left(\Delta S_{10}+\Delta S_{20}\right) A_{0}}{\Delta S_{10} \Delta S_{20}}-\frac{\Delta S_{20} A_{1}}{4 \Delta S_{10} \Delta S_{21}} \sqrt{\left|\frac{4 \Delta S_{21}}{\Delta S_{20}}\right|}-\frac{\Delta S_{10} A_{2}}{4 \Delta S_{20} \Delta S_{12}} \sqrt{\left|\frac{4 \Delta S_{12}}{\Delta S_{10}}\right|}\right] \\
& \alpha_{2}=\frac{l|\tilde{\varepsilon}|^{3}}{4}\left[\frac{A_{0}}{\Delta S_{10} \Delta S_{20}}+\frac{A_{1}}{4 \Delta S_{12} \Delta S_{10}} \sqrt{\left|\frac{4 \Delta S_{21}}{\Delta S_{20}}\right|}+\frac{A_{2}}{4 \Delta S_{21} \Delta S_{20}} \sqrt{\left.\left|\frac{4 \Delta S_{12}}{\Delta S_{10}}\right|\right] .}\right.
\end{aligned}
$$

Equation (9) with definitions (10) is invariant under exchange of the indices 1 and 2. The index $v$ and $\sigma_{\tilde{\varepsilon}}$, the sign of $\tilde{\varepsilon}$, can be determined from the Maslov indices of the periodic orbits. The Maslov index of an unstable real satellite orbit is always $v$, that of a stable real satellite orbit is always $v-\sigma_{\tilde{\varepsilon}}$ and that of the central orbit is $v+\sigma_{\tilde{\varepsilon}}$. The actions of the real orbits are ordered in the same way as their Maslov indices, i.e. $S_{i}>S_{j}$ if and only if $v_{i}>v_{j}$. If both satellite orbits are complex the sign of $\tilde{\varepsilon}$ is given by $\sigma_{\tilde{\varepsilon}}=\operatorname{sign}\left(S_{1}-S_{0}\right)=\operatorname{sign}\left(S_{2}-S_{0}\right)$. 
The coefficients (10) depend only on quantities which also enter the individual contribution (7). As an important consequence, the joint contribution (9) is still invariant under canonical transformations. The transitional approximation is obtained if one keeps only the first of the three pre-exponential terms in the integrand.

There are some special cases of values of $\tilde{a}$ and $\tilde{b}$ for which the integral (9) can be evaluated analytically. They are discussed in appendix B. Numerically useful expressions that can be applied for arbitrary values of $a$ and $b$ are given in appendix C. In the following we discuss different limits of the integral (9). If the action differences $\Delta S_{i j}$ are large in comparison with $\hbar$ then a replacement of the Bessel function by its leading asymptotic term and a stationary-phase evaluation of the integral yields a sum over Gutzwiller contributions (5) for the real satellite orbits. Complex satellites do not contribute since they cannot be reached by a steepest-descent deformation of the integration manifold. (A detailed study of contributions of complex orbits near bifurcations can be found in [16].) This is sensible since the complex satellites, though having complex coordinates, still have real actions and semiclassical amplitudes (cf equations (22) and (24)), and their isolated contributions would not be exponentially suppressed with $\hbar \rightarrow 0$. (In this respect, the role of the complex orbits resembles that of the complex satellite in a period-doubling bifurcation [11].)

The contribution of the central orbit is given by the leading semiclassical contribution from the boundary of the integral at $I=0$.

In the opposite limit $\tilde{\varepsilon}=0$, i.e. at the bifurcation, all action differences vanish. The leading-order semiclassical contribution of equation (9) is then given by

$$
\begin{aligned}
d_{\xi}(E) \approx \frac{T_{0}}{4 l \sqrt{2 \pi^{3} \hbar^{3}|b|}} \operatorname{Re}\left\{\left[K\left(\sqrt{\frac{|b|+a}{2|b|}}\right) \mathrm{e}^{-\mathrm{i} \pi / 4}+K\left(\sqrt{\frac{|b|-a}{2|b|}}\right) \mathrm{e}^{\mathrm{i} \pi / 4}\right]\right. \\
\left.\quad \times \exp \left(\frac{\mathrm{i}}{\hbar} S_{0}-\frac{\mathrm{i} \pi}{2} v\right)\right\}
\end{aligned}
$$

if $|a|<|b|$, and by

$d_{\xi}(E) \approx \frac{T_{0}}{4 l \sqrt{\pi^{3} \hbar^{3}(|a|+|b|)}} K\left(\sqrt{\frac{2|b|}{|a|+|b|}}\right) \cos \left(\frac{S_{0}}{\hbar}-\frac{\pi}{2} \nu-\frac{\pi}{4} \sigma_{a}\right)$

if $|a|>|b| . \quad K(z)$ denotes the complete elliptic integral of the first kind. $T_{0}$ and $S_{0}$ are, respectively, the period and action of the orbits at the bifurcation, $a$ and $b$ are the coefficients in the normal form (7) for $\varepsilon=0$, and $l=r / 4$ is the repetition number of the satellite orbits. The contributions (11) and (12) are by an order $\hbar^{-1 / 2}$ larger than the contribution of an isolated period orbit, i.e. the singularity index of the bifurcation is $\frac{1}{2}$. Although it is not written explicitly, all quantities in (11) and (12) depend on the integer $l$. In detail, $T_{0, l}=l T_{0, l=1}, S_{0, l}=l S_{0, l=1}, v_{l}=l v_{l=1}$ and $a_{l}=l a_{l=1}$ and $b_{l}=l b_{l=1}$. It follows that the amplitude of the contribution at the bifurcation decreases as $l^{-1 / 2}$ with increasing $l$. However, it cannot be expected that this approximation is good for arbitrarily large $l$. For longer periodic orbits bifurcations tend to occur more frequently. It is expected that for larger $l$ there are other bifurcations which interfere with the considered bifurcation.

The formulae of this section can also be applied, with minor modifications, to twodimensional area-preserving maps whose time-evolution is governed by the Floquet operator $F$. These maps correspond to systems with one degree of freedom whose Hamiltonian operator is periodic in time, $H(t+T)=H(t)$, and $F=U(T)$ is the unitary time-evolution operator for one period. The Floquet operator has unimodular eigenvalues $\mathrm{e}^{-\mathrm{i} \phi_{i}}$ with phases $\phi_{i}$ that are called quasi-energies. The quasi-energies can be determined from a knowledge of the traces of powers of $F$. 
For maps the trace formula approximates $\operatorname{Tr} F^{n}$ semiclassically instead of the level density. Furthermore, one has to pay attention to the following differences in comparison with autonomous systems with two degrees of freedom: (i) the orbits which contribute are those with a fixed period $n$, not those with a given energy $E$; (ii) the primitive periods have to be expressed in units of $T$ and thus are integer valued; (iii) the action is not the reduced energy-dependent one, but depends on time (that is, on the number $n$ ); (iv) instead of taking twice the real part, the full complex contribution has to be taken; (v) the results differ by a further factor of $2 \pi \hbar$. by

It follows that the contribution $C_{\xi}^{(n)}$ of a period-quadrupling bifurcation to $\operatorname{Tr} F^{n}$ is given

$$
C_{\xi}^{(n)} \approx \frac{1}{4 l \hbar} \int_{0}^{\infty} \mathrm{d} I\left[n+\alpha_{1} I+\alpha_{2} I^{2}\right] J_{0}\left(\frac{\tilde{b} I^{2}}{\hbar}\right) \exp \left\{\frac{\mathrm{i}}{\hbar}\left(S_{0}-\tilde{\varepsilon} I-\tilde{a} I^{2}\right)-\frac{\mathrm{i} \pi}{2} \nu\right\}
$$

where the quantities $\tilde{a}, \tilde{b}, \tilde{\varepsilon}, \alpha_{1}$ and $\alpha_{2}$ are determined by (10) with $T_{0}=n$ and $A_{i}=n /\left(r_{i} \sqrt{\left.\left|\operatorname{Tr} M_{i}-2\right|\right)}\right.$. Here $r_{i}$ and $M_{i}$ are the repetition number and monodromy matrix of the orbit, respectively.

\section{Numerical results}

We now test the uniform approximation numerically on the example of a periodically kicked top [17-19] and compare the results with those for the transitional approximation (valid close to the bifurcation) and the Gutzwiller approximation (which treats the orbits as being isolated). The kicked top is a dynamical system that involves the angular-momentum operators $J_{x}, J_{y}, J_{z}$ which satisfy the usual commutation relations $\left[J_{k}, J_{l}\right]=\mathrm{i} \epsilon_{k l m} J_{m}$, where $\hbar$ is set to unity. The evolution of the system conserves the total angular momentum $J_{x}^{2}+J_{y}^{2}+J_{z}^{2}=j(j+1)$. This introduces the quantum number $j$ which fixes the dimension $2 j+1$ of the Hilbert space. $j+\frac{1}{2}$ further plays the role of the inverse of Planck's constant, and the semiclassical limit is reached by $j \rightarrow \infty$. After normalization of the angularmomentum vector the phase space of the classical system is revealed as the unit sphere.

The specific top that is considered here is described by the Floquet operator

$F=\exp \left[-\mathrm{i} \frac{k_{z}}{2 j+1} J_{z}^{2}-\mathrm{i} p_{z} J_{z}\right] \exp \left[-\mathrm{i} p_{y} J_{y}\right] \exp \left[-\mathrm{i} \frac{k_{x}}{2 j+1} J_{x}^{2}-\mathrm{i} p_{x} J_{x}\right]$.

This describes rotations by angles $p_{i}$ and nonlinear rotations (torsions) of strength $k_{i}$. For the study of bifurcations we hold the values of the $p_{i}$ fixed $\left(p_{x}=0.3, p_{y}=1.0, p_{z}=0.8\right.$ ) and vary $k=k_{z}=10 k_{x}$ as a control parameter. The classical counterpart of the system is integrable for $k=0$ and displays well developed chaos at $k=5$.

At $k=0$ the top describes a linear rotation. In this situation the system has only two periodic orbits, both of period one and positioned at the intersection of the rotation axis with the spherical phase space. They are called the trivial periodic orbits. As $k$ is increased, new orbits show up in bifurcations. The first two period-quadrupling bifurcations are encountered at $k=k^{(1)}=1.0055 \ldots$ and $k=k^{(2)}=1.1954 \ldots$ Both have one of the trivial orbits in their centre. The next period-quadrupling happens at $k=k^{(3)}=3.0336 \ldots$. The central orbit of this bifurcation is born at a smaller value of $k=k^{(4)}=2.4497 \ldots$ in a tangent bifurcation together with an unstable partner. The form of the three period-quadrupling bifurcations is of type $|a|>|b|$, i.e. on one side of the bifurcation $\left(k<k^{(1,2,3)}\right)$ both satellite orbits are complex and on the other side $\left(k>k^{(1,2,3)}\right)$ both satellites are real.

Contributions of periodic orbits engaged in period-quadrupling bifurcations first show up in the trace $\operatorname{Tr} F^{4}$ since the repetition number of the central orbit has to be a multiple 

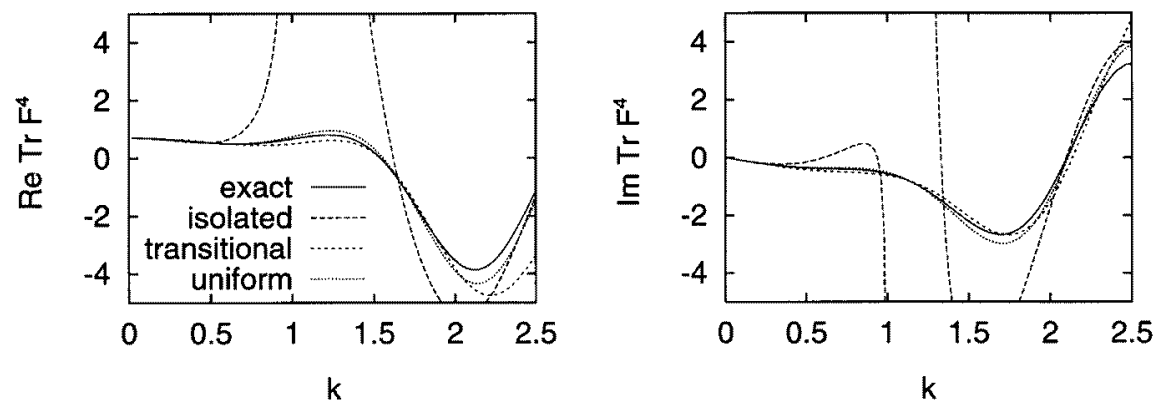

Figure 1. The trace $\operatorname{Tr} F^{4}$ of the fourth power of the Floquet operator (14) as a function of the control parameter $k$ for $j=4$. The plots show the exact quantum result, the uniform approximation, the transitional approximation, and the approximation which considers the orbits as isolated. One observes that the isolated approximation diverges at the period-quadrupling bifurcations while the transitional and the uniform approximation behave regularly there. However, the transitional approximation starts to break down far to the right of the bifurcations.

of four. In the following we describe different semiclassical contributions to $\operatorname{Tr} F^{4}$. In general, orbits of primitive period one, two, and four enter this trace. Complex orbits with the same primitive periods can also contribute, however, they must be reachable by a steepest-descent contour deformation. All these orbits can participate in bifurcations. One has to deal with tangent bifurcations of orbits of primitive period one, two, and four, period-doubling bifurcations with central orbits of primitive period one and two, and period-quadrupling bifurcations with central orbits of primitive period one. For $0 \leqslant k \lesssim 2.5$, however, only a few orbits are relevant, namely, those which participate in the period-quadrupling bifurcations at $k^{(1)}$ and $k^{(2)}$, and in the tangent bifurcation at $k^{(4)}$.

In figure 1 the trace $\operatorname{Tr} F^{4}$ for $j=4$ is plotted against $k$. The exact result is compared with three semiclassical approximations which, respectively, treat the two periodquadrupling bifurcations by the uniform approximation, the transitional approximation, and the approximation that considers the orbits as isolated. The tangent bifurcation is described in all three cases by the uniform approximation of [11]. Observe that the isolated-orbit approximation diverges at the period-quadrupling bifurcations whereas the transitional and the uniform approximation behave regularly there. The transitional approximation, however, loses accuracy for the largest $k$-values in the displayed range, which is most clearly seen in the real part of the trace.

In figure 2 we investigate the behaviour of the semiclassical approximations as the semiclassical limit is approached, i.e. we fix the parameter $k$ at $k=1.5$ and increase the value of $j$. For low values of $j$ the isolated approximation shows large deviations while the transitional and the uniform approximation are accurate. For larger values of $j$ the sum of isolated contributions gains validity, since the effective Planck's constant $1 /(j+1 / 2)$ becomes small in comparison with the action differences of the orbits. The transitional approximation on the other hand becomes slightly more inaccurate, since the error in the semiclassical amplitudes shows up more strongly when the orbits can be considered as isolated. Figure $2(d)$ shows the deviation $\left|\operatorname{Tr} F_{\mathrm{sc}}^{4}-\operatorname{Tr} F_{\mathrm{qm}}^{4}\right|$ of the semiclassical traces $\operatorname{Tr} F_{\mathrm{sc}}^{4}$ from the exact trace $\operatorname{Tr} F_{\mathrm{qm}}^{4}$. This function reveals a crossover between the transitional and the isolated approximation and displays the superiority of the uniform approximation over the whole range of $j$ : the uniform approximation is up to an order of magnitude more accurate than the other two approximations. 

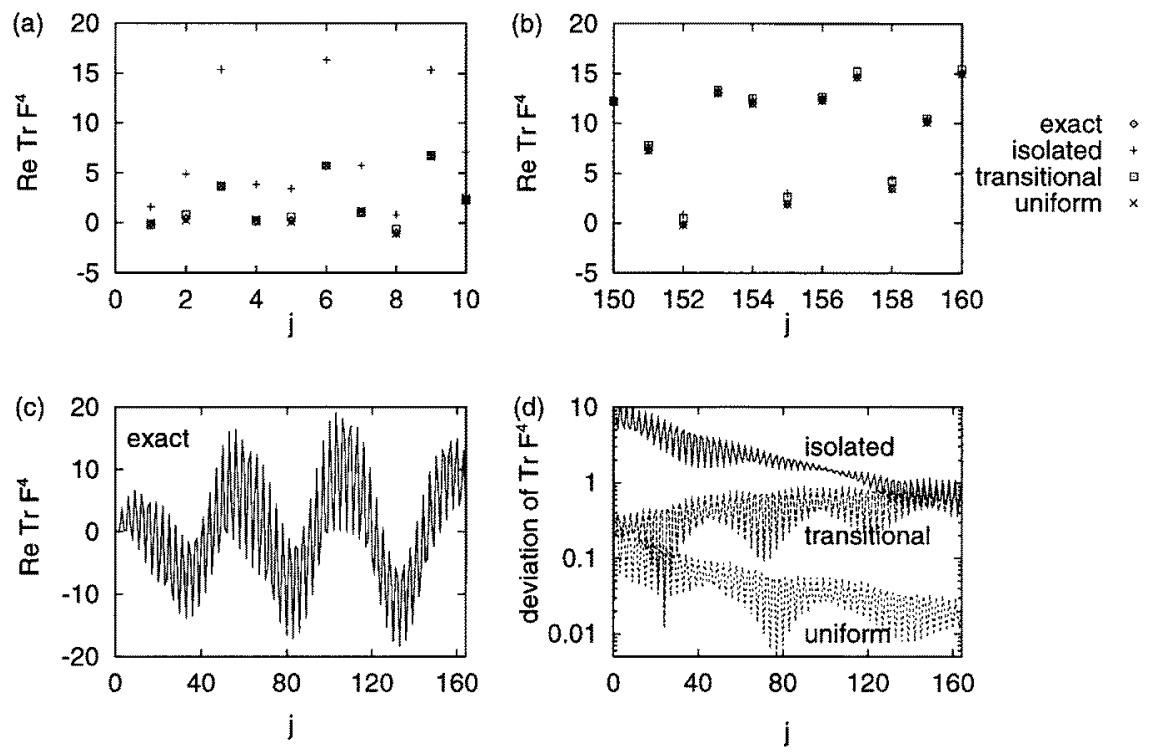

Figure 2. The trace $\operatorname{Tr} F^{4}$ as a function of $j$ for $k=1.5$. (a), (b) The real part of the exact quantum result, the uniform approximation, the transitional approximation, and the approximation which considers the orbits as isolated. $(c)$ Illustration of the oscillations of the exact trace. $(d)$ The error of the semiclassical traces, measured by the absolute value of their deviation from the exact one.

Figure 2(c) illustrates an oscillatory behaviour of $\operatorname{Tr} F^{4}$ which semiclassically originates from the interference of the contributions from the two period-quadrupling bifurcations. These contributions can be separated by considering the function

$$
T^{(n)}(S)=\frac{1}{j_{\max }-j_{\min }+1} \sum_{j=j_{\min }}^{j_{\max }} \mathrm{e}^{-\mathrm{i} j S} \operatorname{Tr} F^{n}(j)
$$

which has peaks at the positions of the actions of the periodic orbits. In its essence this function is a Fourier coefficient of $\operatorname{Tr} F^{n}$ with respect to $j$. It allows us to study the contributions of periodic-orbit clusters individually in the case that the action differences of orbits from different clusters are sufficiently large. A convenient testing tool is then a study of the peak height $T^{(n)}(S)$ at the value of the classical action $S_{\mathrm{cl}}$ of a given orbit in a cluster as a function of $k$ or for different values of $j_{\min }$ and $j_{\max }$ in (15).

In figure 3 we show a quantum-mechanical evaluation of $\left|T^{(4)}\left(S_{\mathrm{cl}}\right)\right|^{2}$ for the three orbits involved in the period-quadrupling at $k^{(1)}$ as the parameter $k$ is steered across the bifurcation. We use $j_{\min }=1$ and $j_{\max }=64$. The exact quantum-mechanical curves are compared with results for the uniform, the transitional, and the isolated approximation. The uniform approximation is excellent over the whole range of $k$. It can hardly be distinguished from the quantum result. The approximation in terms of isolated orbits on the other hand fails completely. It diverges at the bifurcation and only gains validity again for values of $k$ where the amplitudes of the contributions are already quite small. The transitional approximation is good at the bifurcation; however, as $k$ is increased a clear deviation from the quantum result can be seen for the satellite orbits. This result again shows that there is a region where the uniform approximation is essential, since both other approximations, the transitional approximation and the approximation in terms of isolated orbits fail or are inaccurate. 

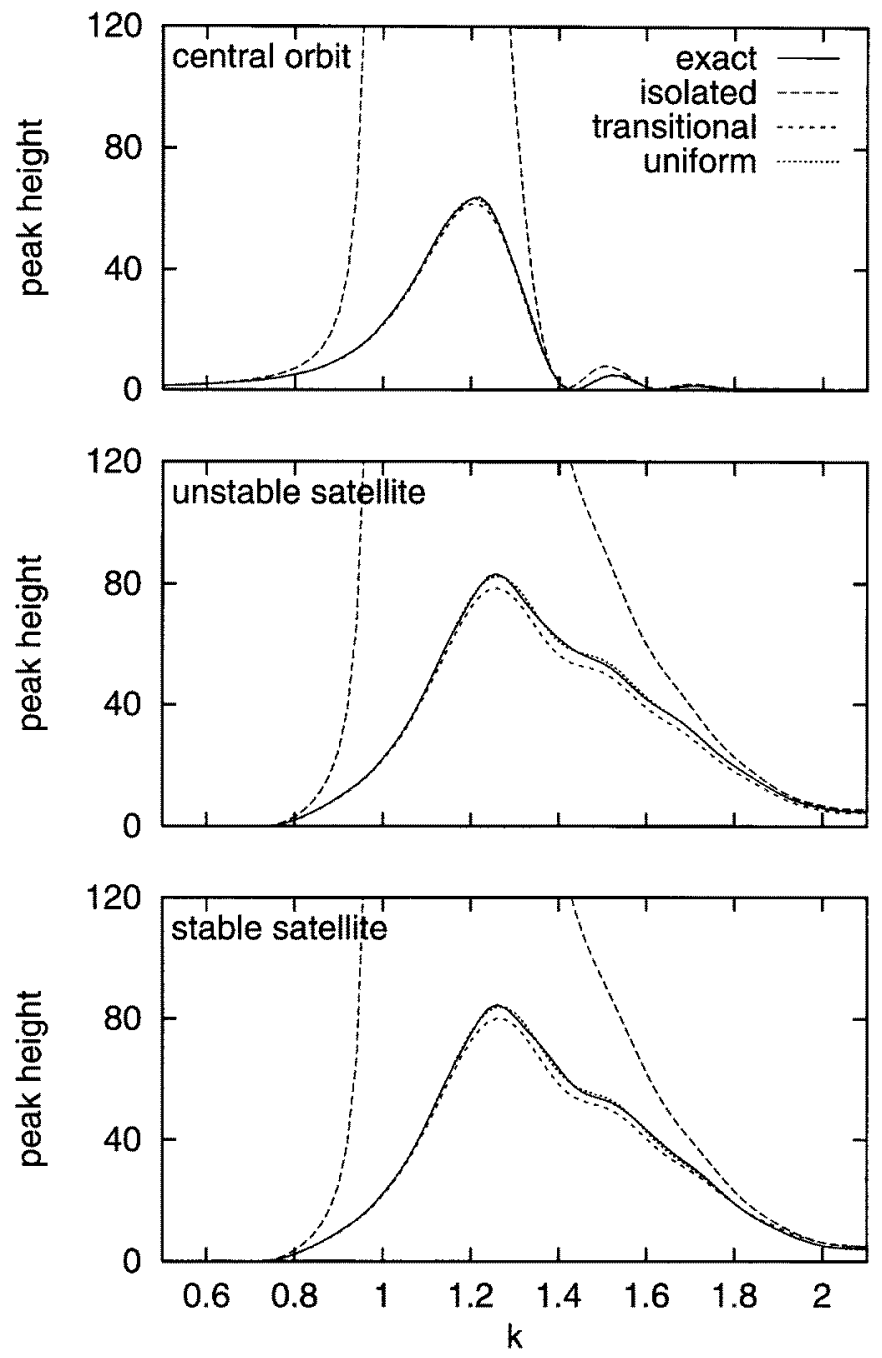

Figure 3. Peak heights $\left|T^{(4)}\left(S_{\mathrm{cl}}(k)\right)\right|^{2}$ at the values of the actions of three periodic orbits as a function of the control parameter $k$. As $k$ goes through $k^{(1)}=1.0055 \ldots$ the three orbits coalesce in a generic period-quadrupling bifurcation. The plots show the exact quantum result, the uniform approximation, the transitional approximation and the approximation which considers the orbits as isolated. They are evaluated with $j_{\min }=1$ and $j_{\max }=64$.

One can also investigate the peak heights for a given $k$ and increasing $j_{\min }$ and $j_{\max }$. In figure 4 the result is shown as a function of $j_{\min }$ with $j_{\max }=j_{\min }+63$. Again, $k$ is set to 1.5. Deviations are visible for the transitional and the isolated approximation. Once more a crossover in the accuracies of these approximations is observed. The uniform approximation is by far superior over the whole range of $j_{\min }$ and cannot be distinguished from the exact result in the plots for the peak heights.

Our numerical studies reveal that the approximation with isolated contributions is valid only far away from bifurcations or for sufficiently small values of Planck's constant while the transitional approximation does not gain validity in the semiclassical limit since it does not involve the correct semiclassical amplitudes. The uniform approximation, however, 

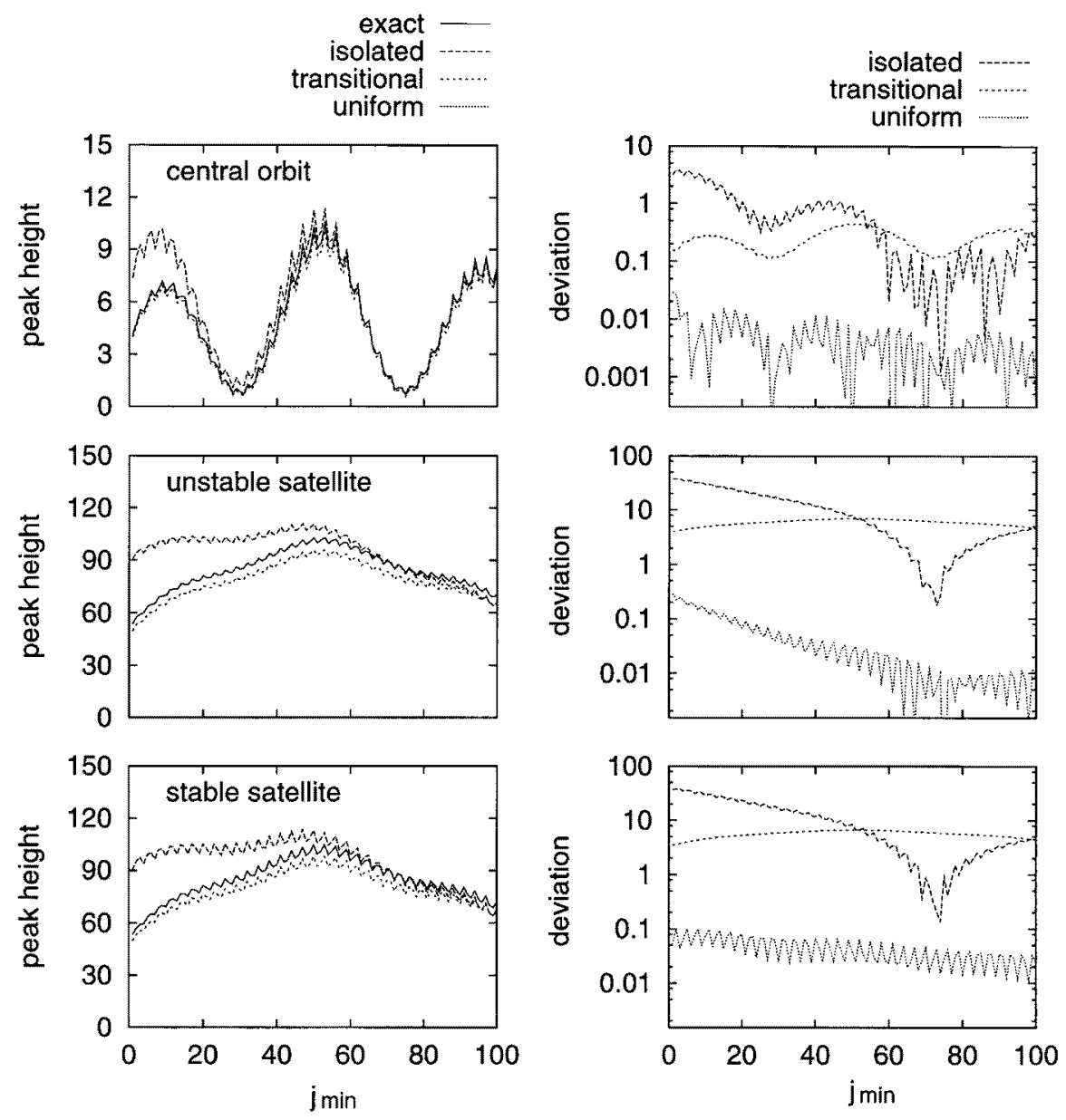

Figure 4. Peak heights $\left|T^{(4)}\left(S_{\mathrm{cl}}\right)\right|^{2}$ at the values of the actions of three periodic orbits as a function of $j_{\min }$ with $j_{\max }=j_{\min }+63$ and $k=1.5$. The plots on the left show the exact quantum result, the uniform approximation, the transitional approximation, and the approximation which considers the orbits as isolated. The plots on the right show the deviation between the semiclassical and the exact peak heights.

gives reliable predictions both close to a bifurcation as well as far away from it, and its accuracy increases in the semiclassical limit.

\section{Conclusions}

In this paper we derived a uniform approximation for the joint contribution of periodic orbits that are involved in a generic period-quadrupling bifurcation. Together with the results of $[10,11]$ this completes the uniform treatment of generic bifurcations in autonomous systems with two degrees of freedom and two-dimensional area-preserving maps. Generic here means that these bifurcations are typically encountered in mixed systems without symmetries as one parameter of the system is varied. They are also called bifurcations of codimension one since only one parameter needs to be changed in order to bring the 
participating orbits into coalescence. In systems with symmetries there can be additional kinds of bifurcations. Some of them can be described by small modifications of the formulae for generic bifurcations [11].

In the following we discuss the limits of validity of the uniform approximations and possible extensions. Although in a generic situation one does not encounter any other form of coalescence of periodic orbits than those discussed above, there are still cases in which the uniform approximations for generic bifurcations have to be modified. The reason for this is that periodic orbits can undergo several subsequent bifurcations. One observes for instance that the iteration of the map corresponding to a normal form describes more periodic orbits of longer periods and allows for additional bifurcations. Another source of additional periodic orbits and bifurcations are higher-order terms in extended normal forms. If the bifurcations occur rapidly one after the other they cannot be considered separately, and instead all participating orbits have to be treated collectively. The next step beyond the isolated treatment of a bifurcation is the collective treatment of two subsequent bifurcations. Often the two consecutive bifurcations can also be considered as being part of a bifurcation of codimension two, since the two bifurcations can be brought into coalescence by varying a second parameter. A collective treatment of two subsequent bifurcations is then necessary if one is sufficiently close to a bifurcation of codimension two in parameter space. The methods for obtaining uniform approximations for these cases are, in principle, the same as for bifurcations of codimension one; however, the normal forms are more complicated. Examples for these normal forms are given in [20, 21], and uniform approximations for bifurcations of codimension two can be found in [22, 23]. It is even possible to completely semiclassically quantize a kicked top with low-dimensional Hilbert space by including uniform approximations for bifurcations of codimension two [24].

As longer and longer periodic orbits are considered, bifurcations tend to occur more rapidly and it is expected that then bifurcations of even higher codimensions become important. This makes the semiclassical treatment of mixed systems more and more complicated. Applications of semiclassical and uniform approximations in mixed systems are therefore most useful in cases where mainly the shortest periodic orbits of a system are needed, for example if one is interested in long-range fluctuations in a spectrum or if the contributions of long periodic orbits are suppressed. Applications of this kind are discussed, for example in [25].

\section{Acknowledgments}

MS wishes to acknowledge financial support by the Deutsche Forschungsgemeinschaft under contract nos DFG-Ste 241/6-1 and /7-2. HS gratefully acknowledges support by the Sonderforschungsbereich 'Unordnung und große Fluktuationen' of the Deutsche Forschungsgemeinschaft.

\section{Appendix A. Derivation of the uniform approximation}

We follow in this section a method from catastrophe theory for obtaining a uniform approximation for an oscillatory integral with nearly coincident stationary points. By this method the exponent of an oscillating integrand is mapped onto a standard normal form with the same structure of stationary points. A description of the method and references to previous work can be found in $[14,15]$. A rigorous treatment including higher-order correction terms is given in [13]. 
The contributions of the periodic orbits to the level density are contained in the integral (2)

$$
d_{\xi}(E) \approx \frac{1}{2 \pi^{2} \hbar^{2} r} \operatorname{Re} \int_{-\infty}^{\infty} \mathrm{d} q^{\prime} \int_{-\infty}^{\infty} \mathrm{d} p g\left(q^{\prime}, p\right) \exp \left[\frac{\mathrm{i}}{\hbar} f\left(q^{\prime}, p\right)-\frac{\mathrm{i} \pi}{2} v\right]
$$

where

$$
f\left(q^{\prime}, p\right)=\hat{S}\left(q^{\prime}, p, E\right)-q^{\prime} p
$$

and

$$
g\left(q^{\prime}, p\right)=\frac{\partial \hat{S}}{\partial E} \sqrt{\frac{\partial^{2} \hat{S}}{\partial p \partial q^{\prime}}} .
$$

The absolute sign of the mixed derivative of $\hat{S}$ inside the square root has been dropped since it is positive near the bifurcation (as can be seen from the normal form (7)). It changes its sign only at conjugate points where the index $v$ changes as well. By writing it without absolute sign the index $v$ can be kept constant and the additional phase arises instead from the square root when its argument becomes negative. The energy dependence of the functions $f$ and $g$ is not written explicitly.

We consider in the following the contributions of three periodic orbits to (16) that undergo a generic period-quadrupling bifurcation as the energy or an external parameter of the system is varied. We assume that any other stationary points of the exponent in (16) which correspond to different periodic orbits are well separated from the stationary points that correspond to the periodic orbits which participate in the bifurcation. This means, for example, that the energy or other parameters of the system have to be limited to ranges in which the orbits do not participate in any further bifurcation. Under these conditions a uniform approximation is derived for the joint contribution of the three periodic orbits.

Near the bifurcation the generating function $\hat{S}\left(q^{\prime}, p, E\right)$ in (17) is approximately given by the normal form (7). From this normal form one can obtain properties of the periodic orbits as is done in the following. First the stationary points of (17) have to be determined. There is one stationary point at the origin which corresponds to the central periodic orbit. The other stationary points are determined conveniently in terms of canonical polar coordinates $I$, $\Phi$ with $p=\sqrt{2 I} \cos \Phi, q^{\prime}=\sqrt{2 I} \sin \Phi$. In terms of these coordinates the normal form is given by

$$
f\left(q^{\prime}(I, \Phi), p(I, \Phi)\right)=S_{0}-\varepsilon I-a I^{2}-b I^{2} \cos (4 \Phi)
$$

and the stationary points of $f$ are determined by the equations

$$
0=\sin (4 \Phi) \quad 0=-\varepsilon-2[a+b \cos (4 \Phi)] I .
$$

There are altogether eight solutions for (20), four with $\cos (4 \Phi)=1$ corresponding to the satellite orbit which is labelled by 1 in the following, and four with $\cos (4 \Phi)=-1$ corresponding to the orbit labelled by 2 . The values of $I$ at the stationary points follow as

$$
I_{1,2}=-\frac{\varepsilon}{2\left(a+\sigma_{1,2} b\right)}
$$

where $\sigma_{1}=1$ and $\sigma_{2}=-1$. The satellite orbits are real if $I_{i}$ is positive, i.e. for $\sigma_{\varepsilon}=-\sigma_{c_{i}}$ where $c_{i}=a+\sigma_{i} b$ with $i \in\{1,2\}$, and we abbreviate the sign of a quantity $x$ by $\sigma_{x}$. For negative $I_{i}$ the coordinates $p$ and $q$ become complex. The evaluation of (19) at the stationary points leads to the values of the actions of the two satellite periodic orbits

$$
S_{1,2}=S_{0}+\frac{\varepsilon^{2}}{4\left(a+\sigma_{1,2} b\right)} \text {. }
$$


One can see from (22) that the action difference between any two of the periodic orbits increases proportionally to $\varepsilon^{2}$ for small $\varepsilon$. The traces of the stability matrices can be determined from

$$
\operatorname{Tr} M=\left(\frac{\partial^{2} \hat{S}}{\partial p \partial q^{\prime}}\right)^{-1}\left(1+\frac{\partial^{2} \hat{S}}{\partial p \partial q^{\prime}} \frac{\partial^{2} \hat{S}}{\partial p \partial q^{\prime}}-\frac{\partial^{2} \hat{S}}{\partial p^{2}} \frac{\partial^{2} \hat{S}}{\partial q^{\prime 2}}\right)
$$

which has to be evaluated at the stationary points. It yields

$$
\operatorname{Tr} M_{0}=2-\varepsilon^{2} \quad \operatorname{Tr} M_{1}=2+\frac{8 b}{a+b} \varepsilon^{2} \quad \operatorname{Tr} M_{2}=2-\frac{8 b}{a-b} \varepsilon^{2} .
$$

Both the actions (22) and the traces of the stability matrices (24) are real quantities even for orbits with complex coordinates.

Finally the Maslov indices of the orbits are determined. They are given by $\nu+\left(n_{\mathrm{n}}-n_{\mathrm{p}}\right) / 2$ where $n_{\mathrm{n}}$ and $n_{\mathrm{p}}$ are the number of negative and positive eigenvalues of the matrix of second derivatives of $f$, respectively. They follow as

$$
v_{0}=v+\sigma_{\varepsilon} \quad v_{1,2}=v+\frac{1}{2}\left(\sigma_{c_{1,2}}-\sigma_{1,2} \sigma_{b}\right) .
$$

From (24) it follows that for an unstable satellite orbit $\sigma_{1,2} \sigma_{b}=\sigma_{c_{1,2}}$, whereas for a stable satellite orbit $\sigma_{1,2} \sigma_{b}=-\sigma_{c_{1,2}}$. Using further the condition for real orbits $\sigma_{c_{1,2}}=-\sigma_{\varepsilon}$ one obtains

$$
v_{0}=v+\sigma_{\varepsilon} \quad v_{s}=v-\sigma_{\varepsilon} \quad v_{u}=v
$$

where the indices $u$ and $s$ denote an unstable and stable real satellite orbit, respectively.

We continue now with the evaluation of the uniform approximation. The equations (22) and (24) entail the conditions (8) which hold for $\varepsilon \rightarrow 0$. If the distance to the bifurcation is increased (by changing the energy or a parameter of the system) higher-order corrections to the normal form can no longer be neglected, and the dependence of $\hat{S}\left(q^{\prime}, p, E\right)$ on $q^{\prime}$ and $p$ becomes more complicated. The main step in the derivation of the uniform approximation consists in the application of a coordinate transformation which then brings the exponent in (16) again into the normal form (inside a region in which the stationary points are located)

$$
f\left(q^{\prime}, p\right)=F\left(Q^{\prime}, P\right)
$$

with

$$
F\left(Q^{\prime}, P\right)=S_{0}-\frac{\tilde{\varepsilon}}{2}\left(Q^{\prime 2}+P^{2}\right)-\frac{\tilde{a}}{4}\left(Q^{\prime 4}+2 P^{2} Q^{\prime 2}+P^{4}\right)-\frac{\tilde{b}}{4}\left(Q^{\prime 4}-6 P^{2} Q^{\prime 2}+P^{4}\right) .
$$

The parameters of $F\left(Q^{\prime}, P\right)$ are chosen in such a way that the mapping from $\left(q^{\prime}, p\right)$ to $\left(Q^{\prime}, P\right)$ is one-to-one in a neighbourhood containing the stationary points. This can be achieved by mapping the stationary points $\left(q_{i}^{\prime}, p_{i}\right)$ of the left-hand side of (27) onto the stationary points $\left(Q_{i}^{\prime}, P_{i}\right)$ of the right-hand side of (27), which leads to the following condition

$$
f\left(q_{i}^{\prime}, p_{i}\right)=F\left(Q_{i}^{\prime}, P_{i}\right)
$$

from which the parameters $\tilde{a}$ and $\tilde{b}$ can be determined.

Condition (29) is already fulfilled for the stationary point at the origin which corresponds to the central orbit. For the other stationary points the evaluation of (29) leads back to (22) where the parameters $\varepsilon, a$ and $b$ now carry a tilde. Solving these equations for $\tilde{a}$ and $\tilde{b}$ results in

$$
\tilde{a}=\frac{\tilde{\varepsilon}^{2}\left(\Delta S_{10}+\Delta S_{20}\right)}{16 \Delta S_{10} \Delta S_{20}} \quad \tilde{b}=\frac{\tilde{\varepsilon}^{2} \Delta S_{21}}{16 \Delta S_{10} \Delta S_{20}}
$$


where $\Delta S_{i j}=\left(S_{i}-S_{j}\right) / 2$. Note that the third parameter $\tilde{\varepsilon}$ of the mapping is not fixed. The reason for this is that the form (28) contains one more parameter than is actually needed. By a simple scaling transformation $Q^{\prime} \rightarrow \lambda Q^{\prime}, P \rightarrow \lambda P$ one can change one of the three parameters into \pm 1 . In analogy to (24) we define $\tilde{\varepsilon}$ by

$$
\tilde{\varepsilon}^{2}=2-\operatorname{Tr} M_{0}
$$

which measures the distance to the bifurcation. The sign of $\tilde{\varepsilon}$ is the same as the sign of $\varepsilon$ and can be determined from the Maslov indices (26) of the central orbit and one real satellite orbit (if both satellite orbits are complex then $\sigma_{\tilde{\varepsilon}}=\sigma_{\tilde{a}}$ ). The difference between the quantities with and without tilde is that those without tilde are obtained from a Taylor expansion around the central orbit whereas the quantities with tilde follow from the mapping. For $\tilde{\varepsilon} \rightarrow 0$ the mapping (27) approaches the identity transformation $\left(Q^{\prime}, P\right)=\left(q^{\prime}, p\right)$ and the quantities with tilde approach those without.

The mapping (27) transforms the integral (16) into

$d_{\xi}(E) \approx \frac{1}{2 \pi^{2} \hbar^{2} r} \operatorname{Re} \int_{-\infty}^{\infty} \mathrm{d} Q^{\prime} \int_{-\infty}^{\infty} \mathrm{d} P G\left(Q^{\prime}, P\right) \exp \left[\frac{\mathrm{i}}{\hbar} F\left(Q^{\prime}, P\right)-\frac{\mathrm{i} \pi}{2} v\right]$

where

$$
G\left(Q^{\prime}, P\right)=g\left(q^{\prime}, p\right) \operatorname{det}\left(\frac{\partial\left(q^{\prime}, p\right)}{\partial\left(Q^{\prime}, P\right)}\right)
$$

and the determinant in (33) is the Jacobian of the transformation which will be denoted by $J\left(Q^{\prime}, P\right)$ in the following.

The uniform approximation is obtained by writing the function $G\left(Q^{\prime}, P\right)$ in the following form

$G\left(Q^{\prime}, P\right)=\alpha_{0}-\alpha_{1} \frac{\partial F}{\partial \tilde{\varepsilon}}-\alpha_{2} \frac{\partial F}{\partial \tilde{a}}-\alpha_{3} \frac{\partial F}{\partial \tilde{b}}+H_{1}\left(Q^{\prime}, P\right) \frac{\partial F}{\partial Q^{\prime}}+H_{2}\left(Q^{\prime}, P\right) \frac{\partial F}{\partial P}$.

In order for this representation to be correct, the constants $\alpha_{0}, \ldots, \alpha_{3}$ have to be determined such that the right-hand side of (34) has the correct values at the stationary points. The last two terms in (34) vanish at the stationary points. They can be neglected since after inserting (34) into (32) they lead to terms which are of order $\hbar$ smaller than the other terms as can be seen by an integration by parts. Furthermore, the constant $\alpha_{3}$ can be set equal to zero, since after inserting (34) into (32) the integral proportional to $\alpha_{3}$ can be expressed in terms of the integrals that are proportional to the other $\alpha_{i}$, as can be shown by another integration by parts.

The remaining parameters $\alpha_{0,1,2}$ in (34) are obtained by an evaluation of (33) at the stationary points. For that purpose the value of the Jacobian $J\left(Q^{\prime}, P\right)$ at the stationary points has to be determined. This is done by differentiating (27) twice. With the notation $\left(z_{1}, z_{2}\right):=\left(q^{\prime}, p\right)$ and $\left(Z_{1}, Z_{2}\right):=\left(Q^{\prime}, P\right)$ this results in

$$
\left.\frac{\partial^{2} F}{\partial Z_{k} \partial Z_{l}}\right|_{\substack{Q^{\prime}=Q_{i}^{\prime} \\ P=P_{i}}}=\left.\sum_{m, n=1}^{2} \frac{\partial z_{m}}{\partial Z_{k}} \frac{\partial z_{n}}{\partial Z_{l}} \frac{\partial^{2} f}{\partial z_{m} \partial z_{n}}\right|_{\substack{q^{\prime}=q_{i}^{\prime} \\ p=p_{i}}}
$$

from which the Jacobian $J\left(Q^{\prime}, P\right)$ follows as

$$
J\left(Q^{\prime}, P\right)=\sqrt{\frac{\operatorname{det}\left[\frac{\partial^{2} F}{\partial Z_{i} \partial Z_{j}}\right]}{\operatorname{det}\left[\frac{\partial^{2} f}{\partial z_{k} \partial z_{l}}\right]} .}
$$


By using (23) this result can be expressed in the form

$$
\left[J\left(Q^{\prime}, P\right) \sqrt{\frac{\partial^{2} \hat{S}}{\partial p \partial q^{\prime}}}\right]_{\substack{q^{\prime}=q_{i}^{\prime} \\ p=p_{i}}}=\sqrt{\frac{\operatorname{Tr} \tilde{M}_{i}-2}{\operatorname{Tr} M_{i}-2}}
$$

where we define

$$
\operatorname{Tr} \tilde{M}_{0}=2-\tilde{\varepsilon}^{2} \quad \operatorname{Tr} \tilde{M}_{1}=2+\frac{8 \tilde{b}}{\tilde{a}+\tilde{b}} \tilde{\varepsilon}^{2} \quad \operatorname{Tr} \tilde{M}_{2}=2-\frac{8 \tilde{b}}{\tilde{a}-\tilde{b}} \tilde{\varepsilon}^{2} .
$$

As before the quantities with tilde approach those without as $\tilde{\varepsilon} \rightarrow 0$. We continue now with the determination of the $\alpha_{i}$. By evaluating (33) at the stationary points with (18), (34) and (37) one obtains the following conditions

$$
\alpha_{0}+\alpha_{1} \tilde{I}_{i}+\alpha_{2} \tilde{I}_{i}^{2}=T_{i} \sqrt{\frac{\operatorname{Tr} \tilde{M}_{i}-2}{\operatorname{Tr} M_{i}-2}}
$$

where $\tilde{I}_{1}$ and $\tilde{I}_{2}$ are given by (21) if the parameters on the right-hand side of (21) are given a tilde, and $\tilde{I}_{0}=0$. The constants $\alpha_{0}, \alpha_{1}$ and $\alpha_{2}$ follow from (39) and the definition (31) as

$$
\begin{aligned}
& \alpha_{0}= T_{0} \\
& \alpha_{1}=\frac{4 \tilde{a}}{\tilde{\varepsilon}} \alpha_{0}-\frac{(\tilde{a}+\tilde{b})^{2} T_{1}}{\tilde{b} \tilde{\varepsilon}} \sqrt{\frac{8 \tilde{b} \tilde{\varepsilon}^{2}}{(\tilde{a}+\tilde{b})\left(\operatorname{Tr} M_{1}-2\right)}}+\frac{(\tilde{a}-\tilde{b})^{2} T_{2}}{\tilde{b} \tilde{\varepsilon}} \sqrt{\frac{-8 \tilde{b} \tilde{\varepsilon}^{2}}{(\tilde{a}-\tilde{b})\left(\operatorname{Tr} M_{2}-2\right)}} \\
& \alpha_{2}=\frac{4\left(\tilde{a}^{2}-\tilde{b}^{2}\right)}{\tilde{\varepsilon}^{2}}\left[\alpha_{0}-\frac{(\tilde{a}+\tilde{b}) T_{1}}{2 \tilde{b}} \sqrt{\frac{8 \tilde{b} \tilde{\varepsilon}^{2}}{(\tilde{a}+\tilde{b})\left(\operatorname{Tr} M_{1}-2\right)}}\right. \\
&\left.+\frac{(\tilde{a}-\tilde{b}) T_{2}}{2 \tilde{b}} \sqrt{\frac{-8 \tilde{b} \tilde{\varepsilon}^{2}}{(\tilde{a}-\tilde{b})\left(\operatorname{Tr} M_{2}-2\right)}}\right] .
\end{aligned}
$$

This completely specifies the uniform approximation. The relations (10) for the $\alpha_{i}$ follow from (40) by using (30). The integral representation for the uniform approximation is obtained by changing the integration variables in (32) to canonical polar coordinates $I, \Phi$ with $P=\sqrt{2 I} \cos \Phi, Q^{\prime}=\sqrt{2 I} \sin \Phi$ and performing the integration over $\Phi$. With the approximation for $G\left(Q^{\prime}, P\right)$ that is discussed after (34) this results in the final expression (9).

\section{Appendix B. Special cases}

\section{B.1. The case $S_{0}=\left(S_{1}+S_{2}\right) / 2$}

In the case where the action of the central orbit is the mean of the actions of the satellite orbits, the diffraction integral in (9) can be evaluated analytically since $\tilde{a}=0$. It is a special case of the form of the bifurcation with $|\tilde{a}|<|\tilde{b}|$. The first satellite orbit is real if $\sigma_{\tilde{\varepsilon}}=-\sigma_{\tilde{b}}$ and the second if $\sigma_{\tilde{\varepsilon}}=\sigma_{\tilde{b}}$. We define in the following $\Delta S=\left(S_{1}-S_{0}\right) / 2$. The integral in (9) can be evaluated by the relation

$$
\int_{0}^{\infty} \mathrm{d} I J_{0}\left(I^{2}\right) \exp (-\mathrm{i} \eta I)=\frac{\sqrt{-\mathrm{i} \pi} \eta}{16} H_{1 / 4}^{(1)}\left(\mathrm{e}^{\mathrm{i} \pi\left(\sigma_{\eta}+1\right)} \frac{\eta^{2}}{8}\right) H_{1 / 4}^{(1)}\left(\mathrm{e}^{\mathrm{i} \pi \sigma_{\eta}} \frac{\eta^{2}}{8}\right)
$$


and the first two derivatives of this equation with respect to $\eta$. As before, $\sigma_{\eta}$ denotes the sign of $\eta$. Altogether one obtains the following result for the uniform approximation

$$
\begin{array}{r}
d_{\xi}(E) \approx \frac{|\Delta S|}{2 \hbar^{2}} \operatorname{Re}\left\{\left[\left(\frac{A_{0}}{2}+\frac{A_{1}}{4 \sqrt{2}}+\frac{A_{2}}{4 \sqrt{2}}\right) B_{1}-\mathrm{i} \sigma_{\tilde{b}}\left(\frac{A_{1}}{4 \sqrt{2}}-\frac{A_{2}}{4 \sqrt{2}}\right) B_{2}\right.\right. \\
\left.\left.+\left(\frac{A_{0}}{2}-\frac{A_{1}}{4 \sqrt{2}}-\frac{A_{2}}{4 \sqrt{2}}\right) B_{3}\right] \exp \left(\frac{\mathrm{i}}{\hbar} S_{0}-\frac{\mathrm{i} \pi}{2}\left(v+\sigma_{\tilde{\varepsilon}}\right)\right)\right\}
\end{array}
$$

where

$$
\begin{aligned}
& B_{1}=H_{1 / 4}^{(+)}(z) H_{1 / 4}^{(-)}(z)+\left(1-\mathrm{i} \sigma_{\tilde{\varepsilon}}\right) H_{1 / 4}^{\left(-\sigma_{\tilde{\varepsilon}}\right)}(z) H_{1 / 4}^{\left(-\sigma_{\tilde{\varepsilon}}\right)}(z) \\
& B_{2}=H_{1 / 4}^{(+)}(z) H_{-3 / 4}^{(-)}(z)+H_{-3 / 4}^{(+)}(z) H_{1 / 4}^{(-)}(z)+2\left(1-\mathrm{i} \sigma_{\tilde{\varepsilon}}\right) H_{1 / 4}^{\left(-\sigma_{\tilde{\varepsilon}}\right)}(z) H_{-3 / 4}^{\left(-\sigma_{\tilde{\varepsilon}}\right)}(z) \\
& B_{3}=H_{-3 / 4}^{(+)}(z) H_{-3 / 4}^{(-)}(z)+\left(1-\mathrm{i} \sigma_{\tilde{\varepsilon}}\right) H_{-3 / 4}^{\left(-\sigma_{\tilde{\varepsilon}}\right)}(z) H_{-3 / 4}^{\left(-\sigma_{\tilde{\varepsilon}}\right)}(z)
\end{aligned}
$$

and $z=|\Delta S| / \hbar$. We have chosen the following notation for the Hankel functions: $H_{v}^{(+)}(z)=H_{v}^{(1)}(z)$ and $H_{v}^{(-)}(z)=H_{v}^{(2)}(z)$.

Near the bifurcation the actions of the two satellite orbits and the traces of the monodromy matrices are given by

$$
S_{1}=S_{0}+\frac{\varepsilon^{2}}{4 b} \quad S_{2}=S_{0}-\frac{\varepsilon^{2}}{4 b} \quad \operatorname{Tr} M_{1,2}=2+8 \varepsilon^{2}
$$

and in the limit $\tilde{\varepsilon} \rightarrow 0$ the contribution

$$
d_{\xi}(E) \approx \frac{T_{0}}{16 l \pi^{2} \hbar^{3 / 2}|b|^{1 / 2}} \Gamma^{2}\left(\frac{1}{4}\right) \cos \left(\frac{S_{0}}{\hbar}-\frac{\pi}{2} v\right)
$$

is obtained, which agrees with (11) for $a=0$.

\section{B.2. The case $S_{1}=\left(S_{0}+S_{2}\right) / 2$}

This is again a case where the integral in (9) can be evaluated analytically. This can be seen by considering the previous expression for the uniform approximation (32) where the integration is performed in terms of the $P$ and $Q^{\prime}$ variables. The constants $\tilde{a}$ and $\tilde{b}$ now satisfy the relation $\tilde{a}=3 \tilde{b}$, and the part of the double integral in (32) with constant pre-exponential factor splits into a product of single integrals that can be evaluated. The bifurcation is an example for the case $|\tilde{a}|>|\tilde{b}|$. Both satellite orbits are real if $\sigma_{\tilde{\varepsilon}}=-\sigma_{\tilde{b}}$, and complex if $\sigma_{\tilde{\varepsilon}}=\sigma_{\tilde{b}}$, and we define again $\Delta S=\left(S_{1}-S_{0}\right) / 2$. The part of the integral in (32) with constant pre-exponential factor is given by

$$
\int_{-\infty}^{\infty} \mathrm{d} p \int_{-\infty}^{\infty} \mathrm{d} q^{\prime} \exp \left(-\frac{\mathrm{i} \tilde{\varepsilon}}{2 \hbar}\left(p^{2}+q^{\prime 2}\right)-\frac{\mathrm{i} \tilde{b}}{\hbar}\left(p^{4}+q^{\prime 4}\right)\right)=\frac{2 \pi^{2}|\Delta S| C_{1}^{2}}{|\tilde{\varepsilon}|} \exp \left(\frac{\mathrm{i}}{\hbar} 2 \Delta S\right)
$$

where

$$
C_{1}=J_{-1 / 4}\left(\left|\frac{\Delta S}{\hbar}\right|\right) \mathrm{e}^{-\mathrm{i} \sigma_{\tilde{b}} \pi / 8}-\sigma_{\tilde{b}} \sigma_{\tilde{\varepsilon}} J_{1 / 4}\left(\left|\frac{\Delta S}{\hbar}\right|\right) \mathrm{e}^{\mathrm{i} \sigma_{\tilde{b}} \pi / 8}
$$

and $\sigma_{\tilde{b}}=\operatorname{sign}(\tilde{b})=\operatorname{sign}(\Delta S)$. The terms with $I$ or $I^{2}$ in the exponential prefactor can be obtained from the first two derivatives of (46) with respect to $\tilde{\varepsilon}$.

We further define

$$
C_{2}=J_{3 / 4}\left(\left|\frac{\Delta S}{\hbar}\right|\right) \mathrm{e}^{\mathrm{i} \sigma_{\tilde{b}} 3 \pi / 8}-\sigma_{\tilde{b}} \sigma_{\tilde{\varepsilon}} J_{-3 / 4}\left(\left|\frac{\Delta S}{\hbar}\right|\right) \mathrm{e}^{-\mathrm{i} \sigma_{\tilde{b}} 3 \pi / 8}
$$


and obtain for the uniform approximation (up to higher-order corrections in $\hbar$ )

$$
\begin{gathered}
d_{\xi}(E) \approx \frac{|\Delta S|}{\hbar^{2}} \operatorname{Re}\left\{\left[\left(\frac{A_{0}}{4}+\frac{A_{1}}{4 \sqrt{2}}+\frac{A_{2}}{8}\right) C_{1}^{2}-\left(\frac{A_{0}}{2}-\frac{A_{2}}{4}\right) C_{1} C_{2}\right.\right. \\
\left.\left.+\left(\frac{A_{0}}{4}-\frac{A_{1}}{4 \sqrt{2}}+\frac{A_{2}}{8}\right) C_{2}^{2}\right] \exp \left(\frac{\mathrm{i}}{\hbar} S_{1}-\frac{\mathrm{i} \pi}{2} v\right)\right\} .
\end{gathered}
$$

In the vicinity of the bifurcation the actions of the two satellite orbits and the traces of the monodromy matrices are given by

$$
S_{1}=S_{0}+\frac{\varepsilon^{2}}{16 b} \quad S_{2}=S_{0}+\frac{\varepsilon^{2}}{8 b} \quad \operatorname{Tr} M_{1}=2+2 \varepsilon^{2} \quad \operatorname{Tr} M_{2}=2-4 \varepsilon^{2}
$$

and in the limit $\varepsilon \rightarrow 0$ the following contribution is obtained,

$$
d_{\xi}(E) \approx \frac{T_{0}}{32 l \pi^{2} \hbar^{3 / 2}|b|^{1 / 2}} \Gamma^{2}\left(\frac{1}{4}\right) \cos \left(\frac{S_{0}}{\hbar}-\frac{\pi}{2} v-\frac{\pi}{4} \sigma_{b}\right) .
$$

This agrees with (12) for $a=3 b$.

\section{B.3. Further special cases}

We briefly discuss two additional cases that occur if $\tilde{b}=0$ or $|\tilde{b}|=|\tilde{a}|$.

In the case $\tilde{b}=0$ the actions of the two satellite orbits $S_{1}$ and $S_{2}$ are identical. This can occur in integrable systems where both orbits are part of a torus. During the bifurcation this whole torus of orbits arises. After integrating by parts the formula (9) reduces in the limit $\tilde{b}=0$ to

$$
d_{\xi}(E) \approx \frac{1}{4 l \pi \hbar^{2}} \operatorname{Re} \int_{0}^{\infty} \mathrm{d} I\left[T_{0}+\alpha I\right] \exp \left\{\frac{\mathrm{i}}{\hbar}\left(S_{0}-\tilde{\varepsilon} I-\tilde{a} I^{2}\right)-\frac{\mathrm{i} \pi}{2} \nu\right\}
$$

and thus can be expressed by a Fresnel integral. The constant $\alpha$ is given by

$$
\alpha=\lim _{\tilde{b} \rightarrow 0}\left(\alpha_{1}-\frac{\tilde{\varepsilon}}{2 \tilde{a}} \alpha_{2}\right) .
$$

A discussion of semiclassical approximations for bifurcations in which a torus arises from a stable orbit is given in [26].

The other case $|\tilde{b}|=|\tilde{a}|$ separates the two forms in which the generic period-quadrupling bifurcation can occur. The set of stationary points in the normal form (28) corresponding to one of the two satellite orbits goes to infinity as $\tilde{b}$ approaches $\pm \tilde{a}$. This normal form is not appropriate for a description of this case and correction terms have to be added to it.

\section{Appendix C. Series expansion of the uniform approximation}

The uniform approximation (9) consists of three integrals of type

$$
I_{v} \equiv \int_{0}^{\infty} x^{v} \exp \left[-\mathrm{i} \gamma x-\mathrm{i} \alpha x^{2}\right] J_{0}\left(x^{2}\right) \mathrm{d} x
$$

with $v=0,1,2$. We now present power series in the coefficients $\alpha$ and $\gamma$ that are useful for a numerical evaluation of these integrals. In the case $|\alpha|>1$ (which corresponds to $|b / a|<1$ in the normal form) one expands the integrand around $\gamma=0$ and uses the analytic 
continuation of the integral 6.621.1 in [27]. Expressing a hypergeometric function by its defining series and applying the duplication formula of the gamma function one arrives at

$$
I_{v}=\frac{1}{2} \sum_{m=0}^{\infty} \sum_{n=0}^{\infty} \frac{(\gamma / \mathrm{i})^{n}}{(\mathrm{i} \alpha)^{\frac{n+v+1}{2}}}\left(\frac{1}{2 \alpha}\right)^{2 m} \frac{\Gamma\left(\frac{n+v+1}{2}+2 m\right)}{n !(m !)^{2}} .
$$

For $|\alpha|<1$, i.e. $|b / a|>1$ in the normal form, one uses the integrals 6.699 .1 and 6.699.2 in [27]. Formally, a small imaginary part has to be added to the coefficients in order to assure convergence. At the end this imaginary part is sent to zero. After some transformations similar to those described above one arrives at

$$
\begin{gathered}
I_{\nu}=\frac{\sqrt{2^{v}}}{\sqrt{8 \pi}} \sum_{n, m=0}^{\infty} \frac{(\sqrt{2} \gamma / \mathrm{i})^{n} \alpha^{2 m}}{m ! n !}\left[\sin \left(\frac{1+n+v}{4} \pi\right) \frac{\Gamma^{2}\left(\frac{1+n+v}{4}+m\right)}{\Gamma\left(\frac{1}{2}+m\right)}\right. \\
\left.-\mathrm{i} \alpha \cos \left(\frac{1+n+v}{4} \pi\right) \frac{\Gamma^{2}\left(\frac{3+n+v}{4}+m\right)}{\Gamma\left(\frac{3}{2}+m\right)}\right] .
\end{gathered}
$$

\section{References}

[1] Gutzwiller M C 1971 Periodic orbits and classical quantization conditions J. Math. Phys. 12 343-58

[2] Balian R and Bloch C 1972 Distribution of eigenfrequencies for the wave equation in a finite domain: III. Eigenfrequency density oscillations Ann. Phys. 69 76-160

[3] Berry M V and Tabor M 1976 Closed orbits and the regular bound spectrum Proc. R. Soc. A 349 101-23

[4] Berry M V and Tabor M 1977 Calculating the bound spectrum by path summation in action-angle variables J. Phys. A: Math. Gen. 10 371-9

[5] Gutzwiller M C 1990 Chaos in Classical and Quantum Mechanics (New York: Springer)

[6] Meyer K R 1970 Generic bifurcations of periodic points Trans. Am. Math. Soc. 149 95-107

[7] Brjuno A D 1970 Instability in a Hamiltonian system and the distribution of asteroids Math. USSR Sbornik 12 271-312

[8] Bruno A D 1972 Research on the restricted three body problem. I: Periodic solutions of a Hamiltonian system Preprint No 18 (Moskva: Inst. Prikl. Mat. Akad. Nauk SSSR) 44pp (in Russian)

[9] Ozorio de Almeida A M and Hannay J H 1987 Resonant periodic orbits and the semiclassical energy spectrum J. Phys. A: Math. Gen. 20 5873-83

[10] Sieber M 1996 Uniform approximation for bifurcations of periodic orbits with high repetition numbers $J$. Phys. A: Math. Gen. 29 4715-32

[11] Schomerus H and Sieber M 1997 Bifurcations of periodic orbits and uniform approximations J. Phys. A: Math. Gen. 30 4537-62

[12] Sieber M 1997 Semiclassical transition from an elliptical to an oval billiard J. Phys. A: Math. Gen. 30 4563-96

[13] Duistermaat J J 1974 Oscillatory integrals, Lagrange immersions and unfolding of singularities Commun. Pure Appl. Math. 27 207-81

[14] Connor J N L 1976 Catastrophes and molecular collisions Mol. Phys. 31 33-55

[15] Berry M V 1976 Waves and Thom's theorem Adv. Phys. 25 1-26

[16] Mouchet A 1996 Quelques applications des méthodes semiclassiques en chaos quantique PhD Thesis Université Paris 6

[17] Haake F, Kuś M and Scharf R 1987 Classical and quantum chaos for a kicked top Z. Phys. B 65 381-95

[18] Kuś M, Haake F and Eckhardt B 1993 Quantum effects of periodic orbits for the kicked top Z. Phys. B 92 221-33

[19] Braun P A, Gerwinski P, Haake F and Schomerus H 1996 Semiclassics of rotation and torsion Z. Phys. B $100115-27$

[20] Sadovskií D A, Shaw J A and Delos J B 1995 Organization of sequences of bifurcations of periodic orbits Phys. Rev. Lett. 75 2120-3

[21] Sadovskií D A and Delos J B 1996 Bifurcation of the periodic orbits of Hamiltonian systems: an analysis using normal form theory Phys. Rev. E 54 2033-70

[22] Schomerus H 1997 Semiclassical interference of bifurcations Europhys. Lett. 38 423-8 
[23] Brack M, Reimann S M and Sieber M 1997 Semiclassical interpretation of the mass asymmetry in nuclear fission Phys. Rev. Lett. 79 1817-20

[24] Schomerus H and Haake F 1997 Semiclassical spectra from periodic-orbit clusters in a mixed phase space Phys. Rev. Lett. 79 1022-5

[25] Brack M and Bhaduri R K 1997 Semiclassical Physics (Reading, MA: Addison-Wesley)

[26] Richens P J 1982 On quantisation using periodic classical orbits J. Phys. A: Math. Gen. 15 2101-10

[27] Gradshteyn I S and Ryzhik I M 1994 Table of Integrals, Series, and Products ed A Jeffrey (San Diego, CA: Academic) 